\title{
Anatomical Study of Renal Arteries
}

\author{
Dr. Gollamandala Syamala, M.D ${ }^{1}$, Dr. KSN Prasad, $\mathrm{MS}^{2}$ \\ ${ }^{I}$ (Assistant Professor Anatomy, Siddhartha medical college, Vijayawada, Andhra Pradesh, India) \\ ${ }^{2}$ (Professor \& Head of the Department Anatomy, Siddhartha medical college, Vijayawada, \\ Andhra Pradesh, India)
}

\begin{abstract}
Arterial supply of the kidney is by the Renal artery. These renal arteries are the Antero lateral branches of the abdominal Aorta at the L1 to L2 Inter vertebral disc level. During the dissection of Cadavers in the department of anatomy we observed more than one arterial supply to the kidney and a number of renal arteries showing early ramification before entering into the hilum of kidney. As per our study $70 \%$ of the kidneys are irrigated by single renal artery and $20 \%$ irrigated by the extra renal arteries and $10 \%$ specimens showing early ramification. Variations in renal arterial pattern is more common. So it is useful to have awareness to prevent the surgical hazards because of anatomical consideration.
\end{abstract}

Keywords: Renal artery, Extra Renal artery, Early Ramification, Kidney.

\section{Introduction}

Kidneys are responsible for removing excess water, salts and waste products from the blood and maintaining its PH. To achieve this the blood flow through the wide renal vessels is approximately one quarter of the resting cardiac output. That is 1.2 to 1.4 litres per minute. The renal arteries are large and arises at the upper part of the second lumbar vertebra. The right renal artery passes posterior to the inferior venacava and the renal vein. The left renal artery passes posterior to the left renal vein. The accessory renal artery is not uncommon. Extra renal arteries may arise from the aorta which are precautious origin of segmental artery. The renal artery divides into four or five segmental arteries after passing through the hilum of the kidney. In some specimens it occurs as the early division that is ramification of the main renal artery into segmental branches more proximal to the hilum of the kidney. The renal arteries present great variability in morphological expression regarding their emergence and ramification level. Even though imaging techniques have good resolution, direct anatomical study leads to recognise the vascularity pattern in detail is useful for interpreting surgical approaches and diagnosing the functional alterations.

The main channel of blood supply to a particular morphological element may be expected to vary from on individual to other individual and also in one species of animal to another with respect to variations in its relative size and position. The functionally connected structures inevitably make a simultaneous demand for the circulation and arterial branches supplied to the tissue is from the main source.

The renal arteries present a diameter of around $5 \mathrm{~mm}$. The right renal artery is longer than the left due to the aorta's location to the left of the middle plane of the abdominal cavity. Based on the level of the renal artery ramification, it is known as the hiliar renal artery when it divides at renal hilium level and extrahiliar when the artery emits a segmentary branch entering the kidney's upper or lower poles before entering the hilium (Sampaio \& passos 1992).

\section{Materials and Methods}

Total specimens of 100 are obtained from cadavers placed for dissection in the department of anatomy, Siddhartha medical college, Vijayawada during 2011 to 2015. After clean dissection of specimens exposing the renal arteries from both kidneys right and left we had taken the photos by using digital camera.

\section{Observations}

Out of 100 specimens 70 specimens had the single renal artery passing through the hilum of the kidney. 20 specimens showing the accessory renal arteries, some from the right side and some from the left side going to the upper pole or lower pole of the kidney. 10 specimens showing the early ramification of the renal artery that is before passing through the hilum. Without specification of the side we had taken the parameter as the ramification proximal to the hilum. 


\section{Figures and Tables}

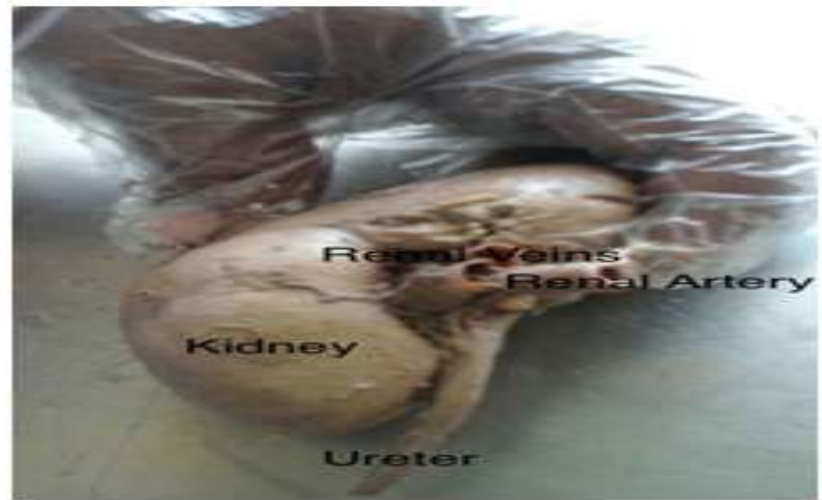

Fig 1: Showing Normal Pattern Single Renal Artery Passing Through Hilum.

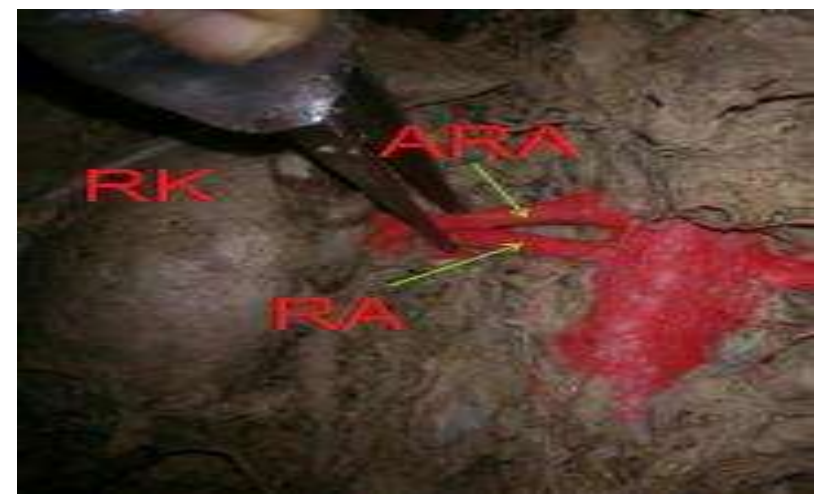

Fig 2: Showing the Accessory Renal Arteries Entering to the Hilum of the Kidney.RK-Right Kidney, RA Renal Artery, ARA - Accessory Renal Artery

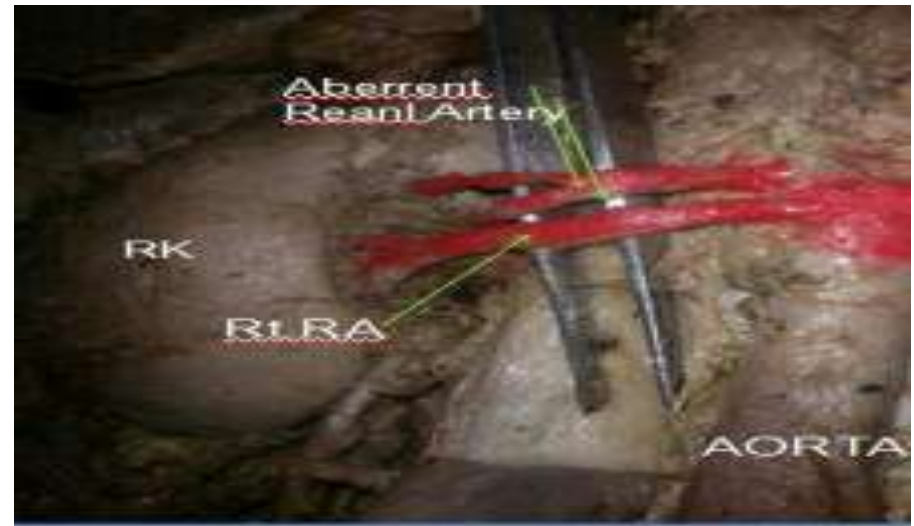

Fig 3: Showing Aberrant Renal Artery Going To The Upper Pole

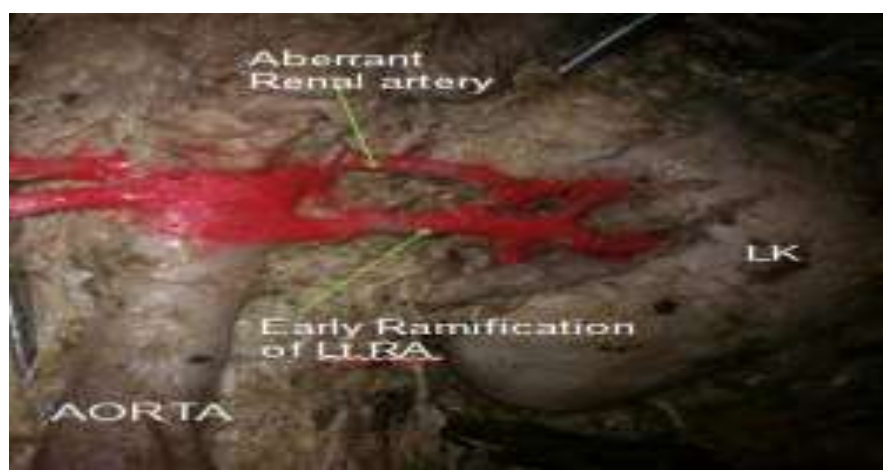

Fig 4: Showing Early Ramification of the Renal Artery 
Table Showing the Observations (number of specimens observed 100)

\begin{tabular}{|l|l|l|l|}
\hline S NO & Pattern of the Renal Artery & No of Specimen Observed & $\%$ \\
\hline 1 & Single Renal Artery & 70 & 70 \\
\hline 2 & $\begin{array}{l}\text { Extra Renal Arteries } \\
\text { a) Accessory or Hilar Renal } \\
\text { Arteries } \\
\text { b) Aberrant Arteries or Polar } \\
\text { Arteries }\end{array}$ & 20 & 20 \\
\hline 3 & Early Ramification & 12 & 8 \\
\hline & Total & 100 & 10 \\
\hline
\end{tabular}

\section{Discussion}

Renal arteries variations are very frequent because of the various developmental positions of the kidney. The extra renal arteries are arising either above or below the main artery. Considerable variations are reported in the literature [1]bordei et al 2004 Double renal arteries originating from the aorta. Surg Radiol Anat, 26: 474-479[2]. $70 \%$ of specimens are supplied by single renal artery agreeing with that of Harrison et al . Similar to that reported by Odman and Ranniger. In the remaining 30\%, 20\% are the extra renal arteries. Out of them $12 \%$ are the accessory renal arteries, $8 \%$ are the aberant renal arteries. Accesssory renal arteries are common due to the persistence of the embryonic arteries which do not regress during the ascent of the kdiney studied by Grays anatomy and Dhar and Lal, 2005[3]. The accessory renal arteries are also seen frequently (Satyapal K (2004), Refer to "Anatomy of renal arterial supply". Clin Anat, 17: 688). They enter the kidney either above or below the hilum (Khamanarong, 2004)[4]. Most of the abnormalities in the renal arteries are due to the various developmental positions of the kidney. As per the reference OzkanU,OguzkurtL,TercanT,OsmanK,KocZ,KocaN (2006)[5] Renal artery origins and variations: angiographic evaluation of 855 consecutive patients. Diagn Interv Radiol, 12: 183-186. With the reference of Sykes D (1963)[9] The arterial supply of the human kidney with special reference to accessory renal arteries. Br J Surg, 50: 368-374.[10] accessorry renal arteries are common.

Renal irrigation is characterized by presenting great variability, the presence of a single bilateral or unilateral renal artery significantly predominating in most studies. Such variability is influenced by ethnic factors, and less so by gender (Ajmani \& Ajmani 1983). The renal artery emerges from the lateral surface of the aorta in most cases (Gracier et al., 2001, Ozan et al., 1997)[6] It emerges from the posterolateral, anterolateral and posterio surfaces with less frequence. Other sites of origin have been described, such as the celiac trunk, common iliac and inferio phrenic artery (Garti et al., 1986). The distance from the renal arteries origin to the superior mesenteric (Garcier et al.,)[8] and celiac trunk (Ajmani \& Ajmani)[7] have been taken as reference in determining the level of emergence. The renal arteries may emerge at the same level, or at different levels, the right renal artery may be found proximal or caudal to the left (Odman \& Ranniger, 2968).

As per my observations, $70 \%$ of the kidney irrigation is by single renal artery. Out of $30 \%, 20 \%$ of the irrigation is by extra renal arteries (accessory or hilar renal arteries 12 and aberant or polar arteries 8) and 10\% early ramification of the articles. As per the references my observations are coinciding and they are obeying the concerning references.

\section{Conclusion}

Proper knowledge of the branching pattern of the vessels is more important specially at the hilum for planning and performing the procedures for the surgeon and urologists. Renal arteries stenosis surgical and radiological techniques. The early division of renal arteries may cause dangers in nephrectomy are partial resection of the kidney. The aim of our effort is to bring some awareness to surgeons above the ramification of the renal arteries at the hilum and multiple renal arteries which are irrigating to the kidney to go for the efficient surgery to decrease the heamarrahgic conditions as the renal artery variations are common. As incidents of renal artery variation is upto $30 \%$.

Great technological advances due to modern diagnostic imaging techniques, particularly in the field of urology, in non-interventionist radiological procedures and in the area of surgery, have emphasized having better morphological references for the renal vascularization patter. Even though imaging techniques have a good resolution, direct Anatomical studies lead to recognizing anatomical vascularization patterns in greater detail, offering a referent having great usefulness for interpreting, managing, surgical approaches and diagnosing functional alterations

Vascular anomalies are usually asymptomatic. The variations observed in this present study, may be functionally, clinically, surgically and radiologically important. The knowledge of this type of variations of renal artery is of atmost important to the surgeons doing kidney transplants and to the radiologists also.

To conclude the awareness of the variations in arteries supplying the organs can also help in minimizing the blood loss during various surgical procedures in this era. 


\section{References}

[1] Double renal arteries originating from the aorta -bordei et al 2004

[2] Surg Radiol Anat, 26: 474-479. 70\% of specimens are supplied by single renal artery (Harrison et al).

[3] Accesssory renal arteries are common due to the persistence of the embryonic arteries - Grays anatomy and Dhar and Lal, 2005

[4] Renal arteries enter the kidney either above or below the hilum - Khamanarong, 2004.

[5] OzkanU,OguzkurtL,TercanT,OsmanK,KocZ,KocaN (2006) Renal artery origins and variations.

[6] The accessory renal arteries are also seen frequently - Satyapal K, 2004

[7] Various levels of emergence of renal arteries - Ajmani \& Ajmani, 1983

[8] The renal artery emerges from the lateral surface of the aorta - Gracier et al., 2001, Ozan et al., 1997.

[9] Renal artery origins and variations Diagn Interv Radiol, 12: 183-186. With the reference of Sykes D (1963)

[10] Accessory renal arteries. Br J Surg, 50: 368-374 